
\title{
25 Research Suare \\ IgE reactivity to fish allergens from Pacific cod (Gadus macrocephalus) in atopic dogs
}

Ichiro Imanishi

Tokyo University of Agriculture and Technology

Jumpei Uchiyama

Azabu University

Keijiro Mizukami

Azabu University

Junichi Kamiie

Azabu University

Keigo Kurata

ITEA Inc

Keita lyori

Vet Derm Tokyo

Masato Fujimura

Fujimura Animal Hospital

Kuniyoshi Shimakura

Tokyo University of Marine Science and Technology

Koji Nishifuji

Tokyo University and Agriculture and Technology

Masahiro Sakaguchi ( $\nabla$ sakagum@azabu-u.ac.jp)

Azabu University https://orcid.org/0000-0002-7594-1943

Research article

Keywords: fish allergy, atopic dermatitis, animal model, immunoglobulin E, allergen component

Posted Date: September 4th, 2020

DOI: https://doi.org/10.21203/rs.2.19058/v4

License: (c) (1) This work is licensed under a Creative Commons Attribution 4.0 International License.

Read Full License 
Version of Record: A version of this preprint was published at BMC Veterinary Research on September 16th, 2020. See the published version at https://doi.org/10.1186/s12917-020-02559-1. 


\section{Abstract}

Background: IgE reactivity to fish allergens in atopic dogs, which are used as models for food allergy, has not been elucidated to date. We investigated IgE reactivity to crude extracts and purified allergens derived from the Pacific cod (Gadus macrocephalus) in atopic dogs to identify the allergenic proteins of cod.

Results: The levels of specific IgE to crude cod extracts were measured in the sera of 179 atopic dogs, including 27 dogs with cod allergy, using enzyme-linked immunosorbent assay (ELISA). Specific IgE to crude cod extracts were present in $36(20 \%)$ of the 179 atopic dogs and in $12(44 \%)$ of the 27 dogs with cod allergy. The allergens in crude cod extracts were analyzed by ELISA, immunoblotting, and liquid chromatography-tandem mass spectrometry. In allergen component analysis, IgE reactivity to tropomyosin and enolase was observed in the sera of dogs with cod allergy. IgE reactivity to parvalbumin, collagen, and tropomyosin was evaluated using the sera of atopic dogs that tested positive for specific $\lg$ E to crude cod extracts. Among the 36 dogs with IgE reactivity to crude cod extracts, 9 (25\%), 14 (39\%), and $18(50 \%)$ dogs tested positive for specific lgE to parvalbumin, collagen, and tropomyosin, respectively.

Conclusions: The IgE reactivity to cod allergens observed in dogs was similar to that in humans, and this finding further supports the use of atopic dogs with fish allergy as a model for fish allergy in humans.

\section{Background}

The prevalence of fish allergy, which affects approximately $0.2 \%$ of the 7.8 billion global population [1], is over ten times higher in geographic regions where fish is an essential dietary component, such as Japan $[2,3]$. Fish allergy is typically known to persist as a life-long condition in contrast to other food allergies [3]. Since clinical cross-reactivity to different fish species is a widely observed characteristic in fish allergy, affected individuals have to compulsorily avoid the consumption of any fish species for extended periods or inadvertent exposure to fish, and the severe or fatal reactions resulting from accidental exposure pose a grave risk for individuals with fish allergy [3]. The components inducing allergic reactions are mostly immunoglobulin (Ig) E-binding proteins, which are referred to as allergens. Among the fish allergens, parvalbumin is the best characterized major allergen that has been detected in several species [4-6]. In a previous study, we identified fish gelatin (type I collagen) as a potential allergen [7]. More recently, other proteins such as tropomyosin, enolases, and aldolases have also been identified as potential fish allergens [8]. Animal models of fish allergy that specifically focus on the management of fish allergenicity will serve as useful tools for improving the quality of life for individuals with fish allergy.

Animal models are beneficial for food allergy testing as they allow rapid and detailed evaluation of allergenicity of certain food products [9]. Canine species are under extensive investigation as useful models in the study of IgE-mediated hypersensitivity as they produce specific IgE to crude extracts and encounter positive oral challenges similar to those observed in human subjects [10-12]. This could be attributed to the fact that similar to that in humans, allergies in canines develop naturally following 
environmental exposure to a broad spectrum of allergens, including those present in food products $[13,14]$. Allergic diseases in canines include atopic dermatitis, gastroenteric inflammation, and anaphylaxis $[15,16]$. Owing to the numerous similarities between canine and human atopic dermatitis [17], atopic dogs have served as animal models for food allergies to cow's milk [11], corn [18], and nuts [12].

The allergens present in food consumed by atopic dogs are assumed to correspond to those in humans [19]. However, Kubota et al. reported that IgE reactivity to allergens in dogs was different from that in humans [20]. To utilize atopic dogs as suitable models for food allergy in humans, it is necessary to analyze the homologies in the IgE reactivity in food allergy between humans and dogs. Cod is one of the most commonly consumed fish species in Europe and Japan [21], and is also the most well-characterized fish species with allergen components [8]. Nevertheless, there is limited information available on IgE reactivity to cod in dogs. Here, we investigated IgE reactivity to crude extracts and purified allergens derived from Pacific cod (Gadus macrocephalus) in atopic dogs.

\section{Results}

\section{Clinical characteristics of atopic dogs with cod allergy}

The study was conducted on 179 atopic dogs of 34 breeds, and there were 79 males and 100 females (age, $3.9 \pm 3.2$ years; range, 2 months -11 years). The elimination diet trials improved the atopic symptoms in 144 dogs (80\%). Among the 31 atopic dogs that underwent cod oral provocation analysis, the clinical symptoms worsened after cod meat exposure in 27 atopic dogs (i.e., dogs with cod allergy). The mean age of dogs with cod allergy was $3.4 \pm 2.6$ years (range, 2 months -8 years), which included 12 males and 15 females. Among these, 8 dogs were administered grilled cod meat and 19 dogs were administered cod-containing dog food. The clinical responses to the provocation test included skin symptoms observed in all dogs (pruritus: 27 , erythema: 16 , and urticaria: 3 ) and concurrent gastrointestinal signs in 3 dogs (11.1\%: vomiting: 3 and diarrhea: 1 ). Among the 27 dogs with cod allergy, eighteen dogs exhibited symptoms within $3 \mathrm{~h}$, whereas 9 dogs exhibited symptoms after a few days. Among the nine dogs with late-onset reaction, one dog exhibited vomiting and diarrhea.

\section{IgE reactivity to crude cod extracts among atopic dogs}

Specific IgE reactivity to crude cod extracts was examined in the atopic dogs using ELISA. Twenty percent $(36 / 179)$ of the dogs had increased levels of specific IgE to crude cod extract (Figure 1). Among the atopic dogs that exhibited positive reactions in the food elimination trials, the levels of specific lgE to crude cod extracts increased in 25\% (36/144). Among the dogs with cod allergy, 44\% (12/27) exhibited positive IgE reactivity to crude cod extracts (Figure 1). All the dogs with late-onset reaction exhibited negative IgE reactivity to crude cod extracts (9/9). Among the four dogs without cod allergy, one dog exhibited false-positive specific IgE reactivity to crude cod extracts. 


\section{IgE reactivity to cod parvalbumin and collagen in atopic dogs exhibiting IgE reactivity to crude cod extracts}

IgE reactivity to the purified cod allergens parvalbumin and collagen was tested in the sera of the 36 dogs with IgE reactivity to crude cod extracts using ELISA. IgE reactivity to parvalbumin was observed in $25 \%$ $(9 / 36)$, whereas IgE reactivity to collagen was observed in $33 \%(12 / 36)$ of the dogs. IgE reactivity to both parvalbumin and collagen was observed in 16\% (6/36) (Figure 5) of the dogs. However, 58\% (21/36) of the dogs did not display IgE reactivity to the purified cod allergens (Figure 2A), which indicates that the reactivity was not specific to fish proteins. We also compared the IgE reactivity to parvalbumin and collagen derived from four fish species (cod, salmon, mackerel, and sardine) in six dogs that reacted to cod parvalbumin and eight dogs that reacted to cod collagen. In these dogs, IgE reactivity to parvalbumin was observed upon reaction with samples derived from cod, salmon, and sardine, while parvalbumin derived from mackerel did not induce a reaction (Figure 2B). Meanwhile, IgE reactivity to collagen derived from all the fish species was observed in $100 \%$ (8/8) of the dogs (Figure $2 \mathrm{C}$ ).

\section{Identification of other cod allergen components in cod allergy in atopic dogs}

Among the 20 atopic dogs with IgE sensitivity to crude cod extracts that did not display reactivity to parvalbumin or collagen, the sera from two dogs (no. 34 and no. 128) were collected, which provided sufficient quantity of sera. While the samples showed reactivity to crude cod extract, no reactivity was observed upon treatment with cod parvalbumin or collagen, as confirmed by the provocation test using cod-containing foods (See Supplementary Table 1, Additional File 1). After the separation of the crude cod extract proteins using SDS-PAGE, IgE-reactive protein bands formed at approximately 35 and $55 \mathrm{kDa}$ were observed in the lgE immunoblotting experiments (Figure 3). LC-MS/MS, which was performed to identify the corresponding proteins in SDS-PAGE, revealed that the protein band of approximately $35 \mathrm{kDa}$ corresponded to tropomyosin derived from golden gray mullet (Liza aurata), and the protein band of approximately $55 \mathrm{kDa}$ corresponded to a enolase derive from python (Python regius) (Table 1).

Table 1. Suspected allergens detected in two dogs with IgE reactivity to crude cod extracts using LC$\mathrm{MS} / \mathrm{MS}$ 


\begin{tabular}{|llllllll|}
\hline Band & $\begin{array}{l}\text { Accession } \\
\text { number }\end{array}$ & $\begin{array}{l}\text { Protein } \\
\text { name/species }\end{array}$ & \multicolumn{3}{l}{ Theoretical } & \multicolumn{3}{l|}{ LC-MS/MS analysis } & \\
\cline { 3 - 7 } & & $\begin{array}{l}\mathrm{MW} \\
(\mathrm{kDa})\end{array}$ & $\mathrm{PI}$ & Score & $\begin{array}{l}\text { Peptide } \\
\text { matches }\end{array}$ & $\begin{array}{l}\% \\
\text { coverage }\end{array}$ \\
\hline 55 & P84335 & $\begin{array}{l}\text { Tropomyosin/ } \\
\text { Liza aurata }\end{array}$ & 32.710 & 4.69 & 111 & 3 & 7 \\
\hline 55 & gi17367183 & $\begin{array}{l}\text { Alpha enolase/ } \\
\text { Python regius }\end{array}$ & 47.541 & 6.97 & 64 & 1 & 2 \\
\hline
\end{tabular}

LC-MS/MS, liquid chromatography-tandem mass spectrometry; MW, molecular weight; PI, isoelectric point.

We next purified cod tropomyosin from the crude cod extract and confirmed its identity using SDS-PAGE (Figure 3). The levels specific IgE to fish tropomyosin in the serum of dog no. 34 were measured using ELISA (See Supplementary Figure 2, Additional File 1); the results revealed that the levels were significantly higher than those in the sera of the 20 control dogs. The levels of specific IgE to tropomyosin derived from other fish species were also higher in dog no. 34 compared to those in the negative controls.

Dog no. 34 presented with specific IgE and a positive reaction in the intradermal test for crude mite extract (House dust mite; Dermatophagoides farinae) (data not shown). We evaluated the levels of specific IgE to recombinant mite tropomyosin (Der f10) in the serum of dog no. 34 using ELISA (See Supplementary Figure 1, Additional File 1) and observed that the levels were significantly higher than those in the sera of the 20 control dogs.

\section{IgE reactivity to cod tropomyosin and crude cod extract in atopic dogs}

Using the sera of 36 atopic dogs with IgE reactivity to cod extract, we determined the IgE reactivity to cod tropomyosin with ELISA (Figure 4), and observed that $50 \%$ (18/36) of the dogs exhibited IgE reactivity to tropomyosin. As shown in Figure 5, 67\% (12/18) of the dogs with atopic dermatitis that had high levels of specific IgE to crude cod extract and tropomyosin did not have specific lgE to cod parvalbumin or collagen. Meanwhile, $25 \%$ (9/36) of the dogs did not display lgE reactivity to any of the tested allergens.

\section{Discussion}

Atopic dermatitis affects approximately $10-20 \%$ of the canine population [22]; hence, the canine atopic dermatitis model could be useful as a spontaneous animal model that can be acquired easily. To assess the potential of atopic dogs with fish allergy as animal models, characterization of the IgE reactivity in 
dogs with cod allergy is useful. We reported the prevalence rate, allergenicity to individual components, and symptomatic association in dogs with cod allergy.

The prevalence of fish allergy might be higher than expected. The rate of food allergy or intolerance to fish has previously been reported to be $1.3 \%(4 / 297)$ in dogs with food allergy or intolerance that was diagnosed by food trials and provocation tests [23]. However, the present study revealed that $20 \%$ (36/179) of the dogs exhibited increased levels of specific lgE to crude cod extract and $25 \%(36 / 144)$ of dogs had food allergy or intolerance, as indicated by the findings of the food elimination trials (Figure 1). Moreover, dogs in Japan might be exposed to fish at a higher frequency than dogs in other countries such as USA. Our field survey on commercial canine dry food products for the estimation of the difference in fish allergen exposure between dogs from Japan and other countries revealed that $75 \%(117 / 157)$ of the Japanese canine dry food products contained fish. In contrast, only 9\% (7/82) of the products produced in Australia and the USA contained fish. These evidences suggest that atopic dogs in Japan might be at a higher risk of developing fish allergy owing to the higher frequency of dietary exposure to fish. These characteristics of atopic dogs might mimic those of humans.

To the best of our knowledge, this is the first study to determine the allergenic potency of parvalbumin and collagen in dogs in terms of their capacity to induce the subsequent production of allergen-specific IgE antibodies. Parvalbumin has a higher allergenic potency than collagen in humans with cod allergy [2]. The present study revealed that the rate of collagen allergy was higher than that of parvalbumin in dogs (Figure 5) and collagen induced a stronger reactivity than that induced by parvalbumin based on the levels of specific IgE in these animals (Figure 2A). This discrepancy might be attributed to the degradation of parvalbumin in dog food during the physical and chemical steps in food processing, since parvalbumin is a water-soluble protein, unlike collagen [4,24]. Additionally, humans exhibit broad crossreactivity to parvalbumin and collagen from distinct fish species $[21,25]$ and the same might occur in dogs with fish allergy as well. The current study suggested that the IgE reactivity to collagen and parvalbumin derived from cod in dogs was comparable to the reactivity to those derived from other fish species such as salmon, mackerel, and sardine (Figure 2B and 2C).

Of note, the rate of tropomyosin allergy was higher than those of parvalbumin or collagen allergies in dogs (Figure 5) and the levels of specific lgE to tropomyosin was higher than those to other allergenic components derived from cod, such as parvalbumin and collagen, in dogs with cod allergy (Figure 2A and 4). Tropomyosin was shown to be a fish allergen in a study using the sera of human patients with tilapia allergy [26]. Additionally, our comparison of the protein sequence of tropomyosin derived from cod to those derived from other commercial fish species revealed a sequence similarity of $94-99 \%$ between the different types of tropomyosin (See Supplementary Table 2, Additional File 1). Tropomyosin is a major allergen that has been attributed in crustacean allergy [27] as well as mite allergy [28] in humans. Although a comparison of the tropomyosin protein sequence from cod and shrimp revealed a low sequence similarity between these (See Supplementary Table 2, Additional File 1), fish-shrimp crossreactivity has been reported previously in humans $[29,30]$. Additionally, mite-crustacean cross-reactivity has been reported widely in humans, and the sequence similarity of tropomyosin derived from the two 
species is over $90 \%$ [31]. In dogs, mites are one of the most frequently sensitizing allergens, and mite tropomyosin is the allergenic component from mites in canine atopic dermatitis [32]. Additionally, in this study, the serum of dog no. 34 elicited reactivity to mite and cod tropomyosin at comparable levels (See Supplementary Figure 1, Additional File 1). Collectively, these findings suggest that mite allergy in dogs with IgE reactivity to mite tropomyosin might be associated with increased IgE reactivity to cod tropomyosin.

The findings of the current study also suggest that enolase could serve as a potential allergen associated with canine fish allergy (Figure 3 and Table 1). Enolase was recently defined as a fish allergen that exhibited cross-reactivity with chicken in humans and dogs [30,33]. Numerous fish proteins, apart from the purified proteins that are recognized as critical allergenic components in humans, have been registered in the International Union of Immunological Societies allergen database [34]. In some cases, minor allergens in humans can act as major allergens in dogs [20,35]. Moreover, in the present study, $25 \%$ $(9 / 36)$ of the dogs with specific IgE to crude cod extracts did not exhibit IgE reactivity to any of the three major purified allergens in ELISA, which implies that other cod allergens be responsible for inducing fish allergy in dogs. Future studies should focus on the identification of other allergens using the sera of atopic docs with IgE reactivity to cod extracts.

Atopic dermatitis with food allergy can be a manifestation of an IgE- or a non-lgE-mediated reaction to food products in humans [36,37]. Similarly, IgE- and non-lgE mediated reactions might also occur in atopic dogs. Approximately $10 \%$ of atopic dogs exhibited positive results in the lymphocyte stimulation test to fish [38,39]. Lymphocyte stimulation tests are used for the diagnosis of non-IgE-mediated food allergies in humans $[40,41]$. Moreover, non-IgE mediated cod allergies may be more common in dogs than in humans. Among dogs with cod allergy, the rate of IgE reactivity to crude cod extracts (44\%; Figure 1 ) was lower than that in humans (90-95\%) [42]. In sixty percent of dogs with cod allergy that did not exhibit IgE reactivity to crude cod extracts, clinical reactions were observed after a few days in the oral provocation tests. Conversely, fish allergy in humans generally presents with the classic symptoms of food allergy shortly after fish intake $[21,42]$. These characteristics of non-IgE mediated reactions in atopic dogs might be independent of those in humans.

\section{Conclusions}

The present study suggests that although IgE reactivities in cod allergy in dogs and humans share some aspects, certain differences remain. Therefore, atopic dogs can serve as animal models of cod allergy if the experiments are conducted with adequate caution. Further analysis would be required to characterize cod allergy in dogs. As this experiment was an illustration, it was not feasible to examine the relationship between the detailed clinical symptoms and responsiveness to each allergen using methods such as specific IgE component analysis. We aim to focus on analyzing the aforementioned relationship in future studies.

\section{Methods}




\section{Sera of atopic dogs and controls}

To examine IgE reactivity in atopic dogs, we collected surplus sera from 179 dogs that were clinically diagnosed with atopic dermatitis from among dogs visiting the Fujimura Animal Hospital (Osaka, Japan) based on the criteria proposed by Willemse [43] and Prelaud et al. [44], which is clinically compatible with the symptoms of atopic dermatitis in humans [17]. Twenty samples collected from laboratory dogs were used as negative controls. The dogs were housed indoors as experimental laboratory animals and did not have prior exposure to fish antigens. None of the laboratory dogs exhibited signs of atopic dermatitis. All sera samples were stored at $-80^{\circ} \mathrm{C}$ until used. Verbal informed consent was provided by the dog owners. All experimental procedures were performed in accordance with Japanese law and were approved by the animal care and user committee of Azabu University.

\section{Food elimination and oral provocation tests}

The elimination diet trials were performed for $6-8$ weeks using commercial hydrolyzed diets on 179 dogs that exhibited clinical symptoms of atopic dermatitis. The dog owners fed commercial hydrolyzed diets that contained ingredients that the dog had no prior exposure to. The ingredients were confirmed based on information provided by the dog owners. When clinical signs were resolved (disappearance of pruritus and hair regrowth), food provocation was performed for one week by administering the original diet. The dogs were checked for clinical symptoms by a veterinary physician every two weeks or once per month. Among the 144 dogs that exhibited clinical improvement in response to the food elimination trials, 31 dogs were observed to exhibit cod reactivity, as evidenced by the oral provocation tests. These dogs were selected based on the diet history, admitted to the animal hospital, and were challenged with various food products containing cod components, including grilled cod meat and cod-containing dog foods (Select Protein Cod and Rice Dry, Royal Canin, Aimargues, France). The provocation trials were conducted by one of the authors (MF), following which the complete resolution of the clinical signs occurred after conducting the food elimination tests. The cod provocation tests were discontinued immediately upon the relapse of the clinical signs that included vomiting, diarrhea, erythema, pruritic, urticaria, and conjunctival hyperemia. We obtained verbal consent for the food elimination trials and provocation tests from the dog owners, as previously described [38]. After the procedures, all dogs were returned to their owners. Supplementary Figure 1, Additional file 1 presents the flow diagram for study participants.

\section{Preparation of crude cod extracts}

Pacific cod was purchased from a fish market in Japan. The fresh, raw meat of four fishes $(500 \mu \mathrm{g})$ was homogenized in $500 \mu \mathrm{l}$ of phosphate-buffered saline (PBS, $10 \mathrm{mM} \mathrm{pH} \mathrm{7.2)} \mathrm{overnight} \mathrm{at} 4{ }^{\circ} \mathrm{C}$ under rotating conditions. After centrifugation at $21500 \times \mathrm{g}$ for $5 \mathrm{~min}$ at $4{ }^{\circ} \mathrm{C}$, the supernatant was collected, and the protein was quantified using the BCA protein assay (Bio-Rad, Hercules, CA, USA). 


\section{Purification of parvalbumin, collagen, and tropomyosin}

Fish parvalbumin [4] and collagen [24] were purified as described previously. Tropomyosin was purified from the freeze-dried powder using Bailey's method with slight modifications [45]. Briefly, freeze-dried fish powder was stirred in a beaker with $75 \mathrm{ml}$ extraction buffer containing $15 \mathrm{mM}$ Tris HCl pH 7.6 (Sigma Aldrich, St Louis, MO, USA), $1 \mathrm{M} \mathrm{KCl}$ (Kanto Kagaku, Tokyo, Japan), and $2 \mathrm{mM}$ dithiothreitol (Sigma Aldrich) overnight at $4{ }^{\circ} \mathrm{C}$. The extract was collected by centrifugation at $5400 \mathrm{xg}$ for $10 \mathrm{~min}$ at $4{ }^{\circ} \mathrm{C}$. The $\mathrm{pH}$ of the supernatant was adjusted to 4.5 using $1 \mathrm{~N} \mathrm{HCl}$ to precipitate tropomyosin, and the precipitate was collected by centrifugation at $5400 \mathrm{xg}$ for $10 \mathrm{~min}$ at $4^{\circ} \mathrm{C}$. The isoelectric precipitation process was repeated once and the precipitate was dissolved in the extraction buffer. The supernatant obtained after the extraction was collected by centrifugation and fractionated using ammonium sulfate to a concentration of $50 \%$. The sample precipitated by ammonium sulfate fractionation was dissolved and dialyzed against PBS. The obtained protein extracts were identified by sodium dodecyl sulfate (SDS)polyacrylamide gel electrophoresis (PAGE).

\section{SDS-PAGE and immunoblotting}

SDS-PAGE was performed according to the method described by Laemmli [46]. Precision plus protein standards (Bio-Rad, Hercules, CA, USA) were used as molecular-mass markers. The crude cod extracts were separated electrophoretically using 5-20\% gradient polyacrylamide gels, and the proteins were visualized either by Coomassie brilliant blue R250 (Bio-Rad) staining or by transferring onto polyvinylidene difluoride membranes (GE Healthcare, Chicago, IL, USA). Immunoblotting was performed as described previously [7]. IgE from patient dog sera were used as primary antibodies, and were diluted at a 1:10 ratio in Tris buffered saline containing $0.1 \%$ Tween-20 and $5 \%$ nonfat dried milk (pH 7.4). Mouse monoclonal anti-dog lgE antibodies $(0.5 \mu \mathrm{g} / \mathrm{ml}$, clone D9) were used as secondary antibodies [47]. Horseradish peroxidase-conjugated goat anti-mouse lgG $(0.05 \mu \mathrm{g} / \mathrm{ml})$ (Bio-Rad Laboratories) were used as tertiary antibodies. An enhanced chemiluminescence immunoblotting detection reagent (GE Healthcare) and the ImageQuant LAS 4000mini (GE Healthcare) were used for detection and visualization, respectively.

\section{Liquid chromatography-tandem mass spectrometry (LC-MS/MS)}

For LC- MS/MS, the protein bands formed after the immunoblotting experiment were excised, and in-gel digestion was performed using $0.5 \mathrm{mg} \mathrm{N}$-tosyl-L-phenylalanine chloromethyl ketone-treated trypsin (Promega, Madison, Wisconsin) at $37^{\circ} \mathrm{C}$ for $16 \mathrm{~h}$. The tryptic digests were acidified using formic acid ( $\mathrm{pH}$ $<2.0$ ) and centrifuged at $21500 \times g$ for $15 \mathrm{~min}$. The supernatants were analyzed using a highperformance liquid chromatograph (Advance System; AMR, Tokyo, Japan) connected to an electrospray 
ionization triple quadrupole mass spectrometer (4000 QTRAP; AB Sciex, Framingham, MA, USA). The extracts were injected into a reversed-phase column (electrospray ionization column [octa decyl silyl]; particle inner diameter, 75 mm; length, 100 mm; diameter $3 \mathrm{~mm}$; LC Assist, Tokyo, Japan) that was eluted with a $5-45 \%$ acetonitrile gradient containing $0.1 \%$ formic acid for $60 \mathrm{~min}$ at $300 \mathrm{nl} / \mathrm{min}$. Ionization was performed at an ion-spray voltage of $2000 \mathrm{~V}$ and a capillary temperature of $200^{\circ} \mathrm{C}$. The mass spectrometer was operated in the positive ion mode between 450-1200 m/z. The MS/MS spectra were obtained in the enhanced production scan mode, and two higher-intensity peaks in each mass spectrometry scan were selected for collision-induced dissociation.

The MS/MS data were used to search the entries under the Liza aurata category in the UniProt database with the Mascot peptide search engine. An MS tolerance of 1.0 Da for precursor ion and an MS/MS tolerance of $0.8 \mathrm{Da}$ were set as the windows of processing parameters for matching the peptide mass values.

\section{Fluorometric enzyme-linked immunosorbent assay (ELISA) for the detection of allergen-specific serum $\lg \mathrm{E}$}

The levels of specific IgE to cod crude extracts and individual purified allergens were measured using fluorometric ELISA, as previously described [20]. A microplate (NUNC Immuno Plate Maxisorp F96; Nalge Nunc International, Roskilde, Denmark) was coated with the crude cod extracts (10 $\mathrm{gg} / \mathrm{ml})$ or the purified allergens (parvalbumin, collagen, or tropomyosin; $1 \mu \mathrm{g} / \mathrm{ml}$ ) and maintained overnight at $4{ }^{\circ} \mathrm{C}$. After washing, the plate was incubated with diluted sera (1:10) in PBS with 10\% (v/v) fetal calf serum and $0.05 \%(v / v)$ Tween 20 at room temperature for $3 \mathrm{~h}$. The plate was then washed and incubated with mouse monoclonal anti-dog IgE antibody $\left(0.5 \mu \mathrm{g} / \mathrm{ml}\right.$, clone D9) overnight at $4{ }^{\circ} \mathrm{C}$ [47]. After washing with PBS containing $0.1 \%(\mathrm{v} / \mathrm{v})$ Tween 20 (PBS-T), the plate was incubated with biotinylated rat monoclonal anti-mouse IgG1 (Zymed Laboratories, San Francisco, CA, USA) for $1 \mathrm{~h}$ at room temperature. After washing, the plate was incubated with b-D-galactosidase-conjugated streptavidin (Zymed Laboratories) for $1 \mathrm{~h}$ at room temperature. After the final wash, the plate was incubated with $0.1 \mathrm{mM} 4$ methylumbelliferyl- b-D-galactopyranoside (Sigma Aldrich) for $2 \mathrm{~h}$ at $37^{\circ} \mathrm{C}$. The enzymatic reaction was terminated by adding $0.1 \mathrm{M}$ glycine- $\mathrm{NaOH}(\mathrm{pH}$ 10.2). The fluorescence intensity was measured in terms of fluorescence units (FU) using a microplate fluorescence reader (Fluoroskan; Flow Laboratories, McLean, VA, USA). The absorbance was measured at $355 \mathrm{~nm}$ using a $460 \mathrm{~nm}$ reference filter. All the washing steps were performed thrice for $5 \mathrm{~min}$ in PBS-T. The cutoff value was determined as the average + three standard deviations (SDs) of FU in the sera from the 20 dogs that served as negative controls in the analysis of the levels of specific IgE to cod crude extracts. ELISA for parvalbumin or collagen from salmon (Atlantic salmon; Salmo salar), sardine (Japanese pilchard; Sardinops melanostictus), and mackerel (Chub mackerel; Scomber japonicus) were performed using individual sera, which ensured that a substantial quantity of sera was available (See Supplementary Figure 1, Additional File 1). All tests were performed in triplicate. 


\section{Abbreviations}

AD Atopic dermatitis

ELISA Enzyme-linked immunosorbent assay

FU Fluorescence units

Ig Immunoglobulin

LC Liquid chromatography

MS/MS Tandem mass spectrometry

PBS Phosphate buffered saline

SD Standard deviation

SDS-PAGE Sodium dodecyl sulfate Poly-acrylamide gel electrophoresis

\section{Declarations}

\section{Ethics approval and consent to participate}

All experimental procedures were carried out in accordance with Japanese law and approved by the animal care and user committee of Azabu University. We obtained verbal consent for the food elimination trials and provocation tests from the dog owners. The ethics committee approved the procedure for verbal consent, because of few disadvantages for dogs and dog owners.

\section{Consent for publication}

Not application.

\section{Availability of data and materials}

All data generated or analyzed during this study are included in this published article and its supplementary information files.

\section{Competing interests}

MS has received research funding from ITEA Inc.; the other authors declare no conflicts of interest.

\section{Funding}

This research was supported by Japan Society for the Promotion of Science (JSPS) KAKENHI Grant Number JP 15K07756, and MEXT supported program for the Private University Research Branding 
Project, 2016-2020, which were received by MS.

\section{Author's contributions}

II made substantial contributions to the study conception and design as well as data acquisition and analysis; he also drafted the article and created all the figures and tables. KK, JK, and MF made substantial contributions to the experiments. JU, KM, KI, KS, and $\mathrm{KN}$ assisted in the study design and manuscript preparation. MS designed the experimental setting, edited the manuscript, and revised the article critically for including important intellectual content. All authors have read and approved the manuscript.

\section{Acknowledgments}

The authors are grateful to Dr. Seiji Kawamoto for providing recombinant mite tropomyosin. We would also like to thank the staff at Enago Group (www.enago.jp) for critically reviewing the draft of this manuscript.

\section{References}

1. Moonesinghe $\mathrm{H}$, Mackenzie $\mathrm{H}$, Venter $\mathrm{C}$, Kilburn S, Turner P, Weir K, et al. Prevalence of fish and shellfish allergy: a systematic review. Ann Allergy Asthma Immunol. 2016;117:264-72.

2. Ebisawa M, Ito K, Fujisawa T. Japanese guidelines for food allergy 2017. Allergol Int. 2017;66:248-64.

3. Sharp MF, Lopata AL. Fish allergy: in review. Clin Rev Allergy Immunol. 2014;46:258-71.

4. Elsayed S, Bennich H. The primary structure of allergen M from cod. Scand J Immunol. 1975;4:203-8.

5. Lindstrom CD, van Do T, Hordvik I, Endresen C, Elsayed S. Cloning of two distinct cDNAs encoding parvalbumin, the major allergen of Atlantic salmon (Salmo salar). Scand J Immunol. 1996;44:33544.

6. Perez-Gordo M, Cuesta-Herranz J, Maroto AS, Cases B, Ibáñez MD, Vivanco F, et al. Identification of sole parvalbumin as a major allergen: study of cross-reactivity between parvalbumins in a spanish fish-allergic population. Clin Exp Allergy. 2011;41:750-8.

7. Sakaguchi M, Toda M, Ebihara T, Irie S, Hori H, Imai A, et al. IgE antibody to fish gelatin (type I collagen) in patients with fish allergy. J Allergy Clin Immunol. 2000;106:579-84.

8. Kuehn A, Swoboda I, Arumugam K, Hilger C, Hentges F. Fish allergens at a glance: variable allergenicity of parvalbumins, the major fish allergens. Front Immunol. 2014;5:179-87.

9. Van Gramberg JL, de Veer MJ, O'Hehir RE, Meeusen ENT, Bischof RJ. Use of animal models to investigate major allergens associated with food allergy. J Allergy (Cairo). 2013;2013:635-45.

10. Santoro D, Marsella R. Animal Models of Allergic Diseases. Vet Sci. 2014;1:192-212.

11. Buchanan BB, Frick OL. The dog as a model for food allergy. Ann Ny Acad. 2002;964:173-83.

12. Teuber SS, del Val G, Morigasaki S, Jung HR, Eisele PH, Frick OL, et al. The atopic dog as a model of peanut and tree nut food allergy. J Allergy Clin Immunol. 2002;110:921-7. 
13. Gedon NKY, Mueller RS. Atopic dermatitis in cats and dogs: a difficult disease for animals and owners. Clin Transl Allergy. 2018;8:41-53.

14. Hensel P, Santoro D, Favrot C, Hill P, Griffin C. Canine atopic dermatitis: detailed guidelines for diagnosis and allergen identification. BMC Vet Res. 2015;11:196.

15. Verlinden A, Hesta M, Millet S, Janssens GP. Food allergy in dogs and cats: a review. Crit Rev Food Sci Nutr. 2006;46:259-73.

16. Olivry T, Mueller RS. Critically appraised topic on adverse food reactions of companion animals (7): signalment and cutaneous manifestations of dogs and cats with adverse food reactions. BMC Vet Res. 2019;15:140.

17. Terada $Y$, Nagata M, Murayama N, Nanko H, Furue M. Clinical comparison of human and canine atopic dermatitis using human diagnostic criteria (Japanese Dermatological Association, 2009): proposal of provisional diagnostic criteria for canine atopic dermatitis. J Dermatol. 2011;38:784-90.

18. Jackson HA, Jackson MW, Coblentz L, Hammerberg B. Evaluation of the clinical and allergen specific serum immunoglobulin $\mathrm{E}$ responses to oral challenge with cornstarch, corn, soy and a soy hydrolysate diet in dogs with spontaneous food allergy. Vet Dermatol. 2003;14:181-7.

19. Mueller RS, Janda J, Jensen-Jarolim E, Rhyner C, Marti E. Allergens in veterinary medicine. Allergy. 2016;71:27-35.

20. Kubota S, Miyaji K, Shimo Y, Shimakura H, Takase Y, Okamoto N, et al. IgE reactivity to a Cry j 3 , an allergen of Japanese cedar (Cryptomeria japonica) pollen in dogs with canine atopic dermatitis. Vet Immunol Immunopathol. 2012;149:132-5.

21. Sharp MF, Lopata AL. Fish allergy: in review. Clin Rev Allergy Immunol. 2014;46:258-71.

22. Chan SK, Leung DYM. Dog and cat allergies: current state of diagnostic approaches and challenges. Allergy Asthma Immunol Res. 2018;10:97-105.

23. Mueller RS, Olivry T, Prelaud P. Critically appraised topic on adverse food reactions of companion animals (2): common food allergen sources in dogs and cats. BMC Vet Res. 2016;12:9.22.

24. Hamada $Y$, Nagashima Y, Shiomi K. Identification of collagen as a new fish allergen. Biosci Biotechnol Biochem. 2001;65:285-91.23.B

25. Kobayashi A, Kobayashi Y, Shiomi K. Fish allergy in patients with parvalbumin-specific immunoglobulin E depends on parvalbumin content rather than molecular differences in the protein among fish species. Biosci Biotechnol Biochem. 2016;80:2018-21.

26. Liu R, Holck AL, Yang E, Liu C, Xue W. Tropomyosin from tilapia (Oreochromis mossambicus) as an allergen. Clin Exp Allergy. 2013;43:365-77.

27. Faber MA, Pascal M, El Kharbouchi O, Sabato V, Hagendorens MM, Decuyper II, et al. Shellfish allergens: tropomyosin and beyond. Allergy. 2017;72:842-8.

28. Asturias JA, Arilla MC, Gomez-Bayon N, Martinez A, Martinez J, Palacios R. Sequencing and high level expression in Escherichia coli of the tropomyosin allergen (Der $p$ 10) from Dermatophagoides pteronyssinus. Biochim Biophys Acta. 1998;1397:27-30. 
29. Kuehn A, Codreanu-Morel F, Lehners-Weber C, Doyen V, Gomez-André SA, Bienvenu F, et al. Crossreactivity to fish and chicken meat - a new clinical syndrome. Allergy. 2016;71:1772-81.

30. Peixoto S, Monteiro T, Carvalho M, Santos M, Matos C, Bartolomé B, et al. Vertebrate tropomyosin as an allergen. J Investig Allergol Clin Immunol. 2018;28:51-3.

31. Wong $\mathrm{L}$, Huang $\mathrm{CH}$, Lee BW. Shellfish and house dust mite allergies: Is the link tropomyosin? Allergy Asthma Immunol Res. 2016;8:101-6.

32. McCall C, Hunter S, Stedman K, Weber E, Hillier A, Bozic C, et al. Characterization and cloning of a major high molecular weight house dust mite allergen (Der $f$ 15) for dogs. Vet Immunol Immunopathol. 2001;78:231-47.

33. Bexley J, Kingswell N, Olivry T. Serum IgE cross-reactivity between fish and chicken meats in dogs. Vet Dermatol. 2018;30:25-8.

34. Pomes A, Davies JM, Gadermaier G, Hilger C, Holzhauser T, Lidholm J, et al. WHO IUIS Allergen Nomenclature Sub-Committee WHO/IUIS Allergen Nomenclature: Providing a common language. Mol Immunol. 2018;100:3-13.

35. Shimakura H, Uchiyama J, Saito T, Miyaji K, Fujimura M, Masuda K, et al. IgE reactivity to hen egg white allergens in dogs with cutaneous adverse food reactions. Vet Immunol Immunopathol. 2016;177:52-7.

36. Spergel JM. Nonimmunoglobulin E-mediated immune reactions to foods. Allergy Asthma Clin Immunol. 2006;2:78-85.

37. Werfel T, Allam J-P, Biedermann T, Eyerich K, Gilles S, Guttman-Yassky E, et al. Cellular and molecular immunologic mechanisms in patients with atopic dermatitis. J Allergy Clin Immunol. 2016;138:33649.26.

38. Fujimura M, Masuda S, Hayashiya M, Okayama T. Flow cytometric analysis of lymphocyte proliferative responses to food allergens in dogs with food allergy. J Vet Med Sci. 2011;73:1309-17.

39. Suto A, Suto Y, Onohara N, Tomizawa Y, Yamamoto-Sugawara Y, Okayama T, et al. Food allergens inducing a lymphocyte-mediated immunological reaction in canine atopic-like dermatitis. $\mathrm{J}$ Vet Med Sci. 2015;77:251-4.

40. Kimura M, Oh S, Narabayashi S, Taguchi T. Usefulness of lymphocyte stimulation test for the diagnosis of intestinal cow's milk allergy in infants. Int Arch Allergy Immunol. 2012;157:58-64.

41. Yagi $H$, Takizawa $T$, Sato $K$, Inoue $T$, Nishida $Y$, Ishige $T$, et al. Severity scales of non-IgE-mediated gastrointestinal food allergies in neonates and infants. Allergol Int. 2019;68:178-84.

42. Matricardi PM, Kleine-Tebbe J, Hoffmann HJ, Valenta R, Hilger C, Hofmaier S, et al. EAACI Molecular Allergology User's Guide. Pediatr Allergy Immunol. 2016;23:1-250.27. Laemmli U. Cleavage of structural proteins during the assembly of the head of bacteriophage T4. Nature. 1970;15:680-5.

43. Willemse T. Atopic dermatitis in dogs. symptomatology and diagnosis. Tierarztl Prax. 1991;19:96101. 
44. Prelaud P, Guaguere E, Alhaidari Z, Faivre, N. Reevaluation of diagnostic criteria of canine atopic dermatitis. Rev Méd Vét. 1998;149:1057-64.

45. Bailey K. Tropomyosin: a new asymmetric protein component of the muscle fibril. Biochemical Journal. 1948;43:271-9.

46. Laemmli U. Cleavage of structural proteins during the assembly of the head of bacteriophage T4. Nature. 1970;15:680-5.47.

47. DeBoer DJ, Ewing KM, Schultz KT. Production and characterization of mouse monoclonal antibodies directed against canine IgE and IgG. Vet Immunol Immunopathol. 1993;37:183-99.

\section{Figures}




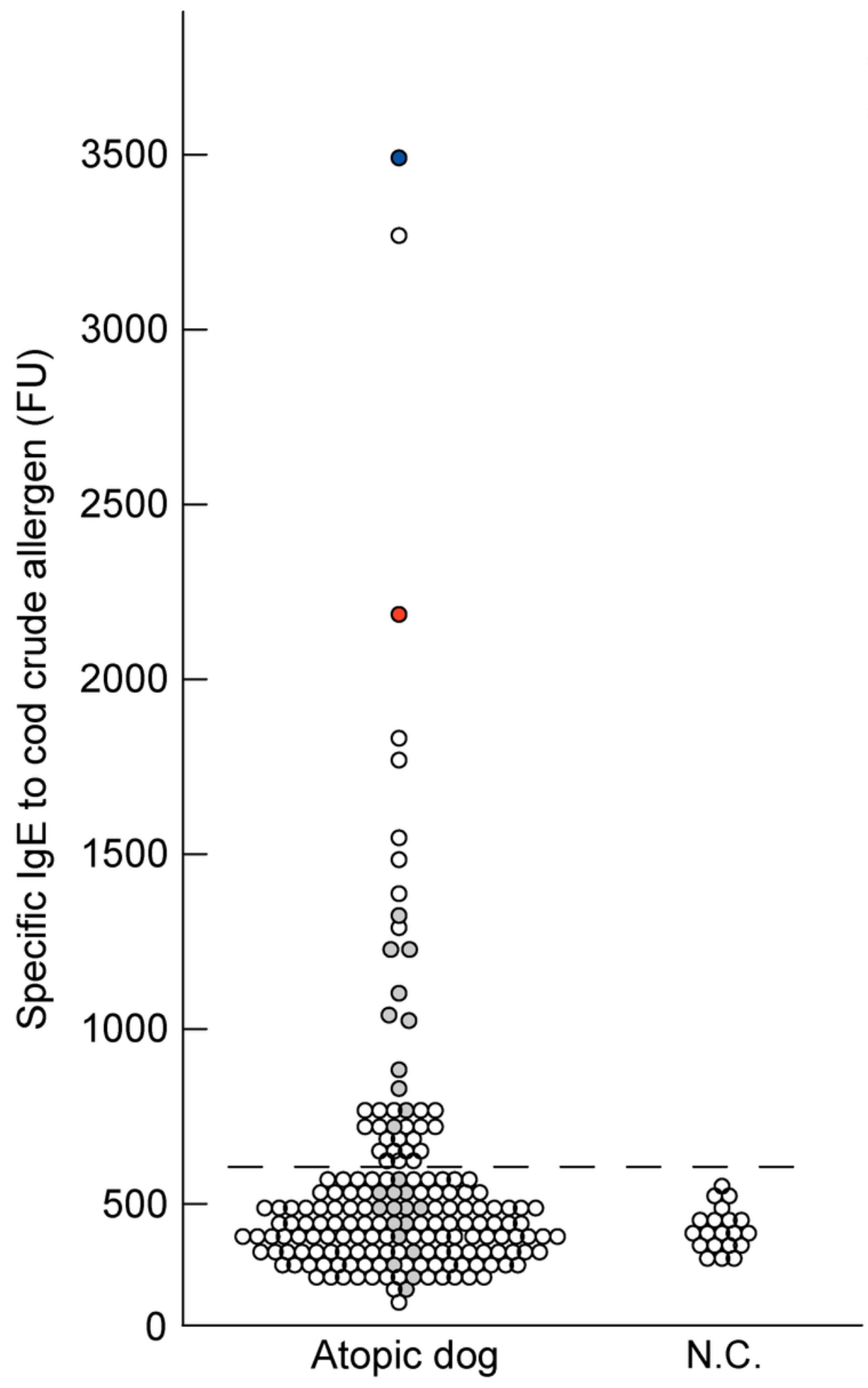

\section{Figure 1}

$\lg E$ reactivity to cod crude extracts in atopic dogs. Based on the levels of specific $\lg E$ to crude cod extracts in 20 negative controls, the cutoff value (mean +3 standard deviation (SD)) was 638 fluorescence units (FU), as indicated by the dotted line. The mean FU \pm SD value of IgE reactivity to crude cod extracts in 179 atopic dogs was $457 \pm 61 \mathrm{FU}$. Thirty-six atopic dogs exhibited specific lgE reactivity to crude cod meat, with IgE levels ranging from 640 to $3483 \mathrm{FU}$. The blue circle indicates IgE reactivity in 
dog no. 34. The red circle indicates IgE reactivity in dog no. 128. The gray circles indicate IgE reactivity in other dogs with cod allergy. IgE, immunoglobulin $\mathrm{E}$.

A)

A)

B)

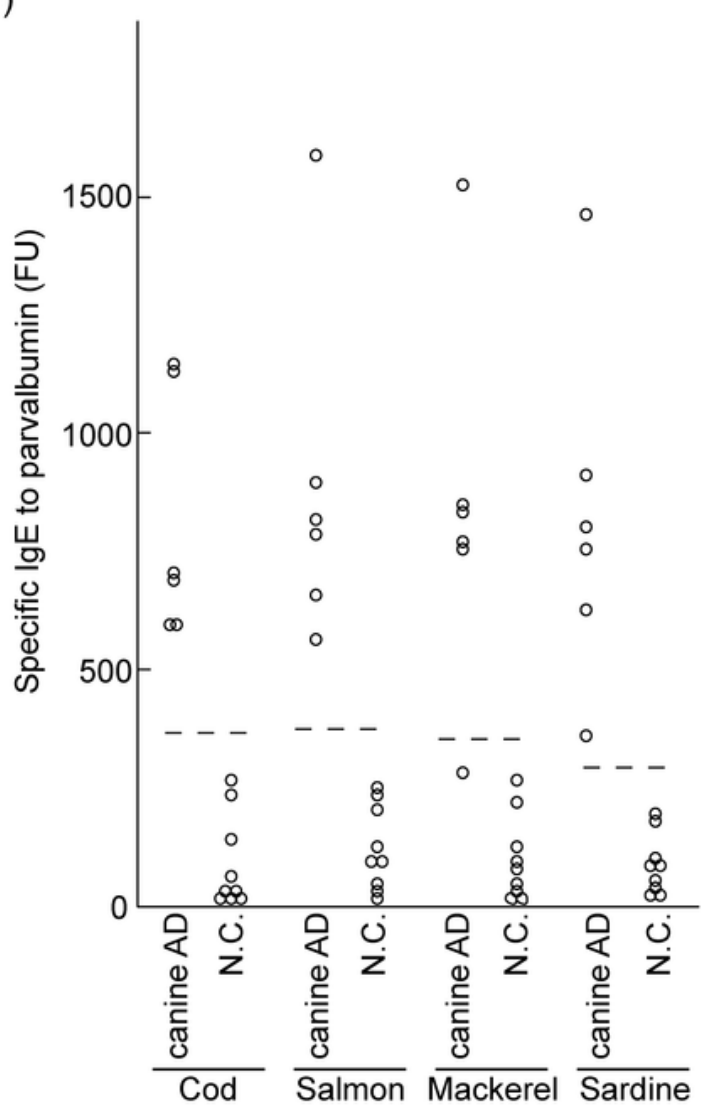

$\circ$

- no. 34 dog

- no. 128 dog
C)

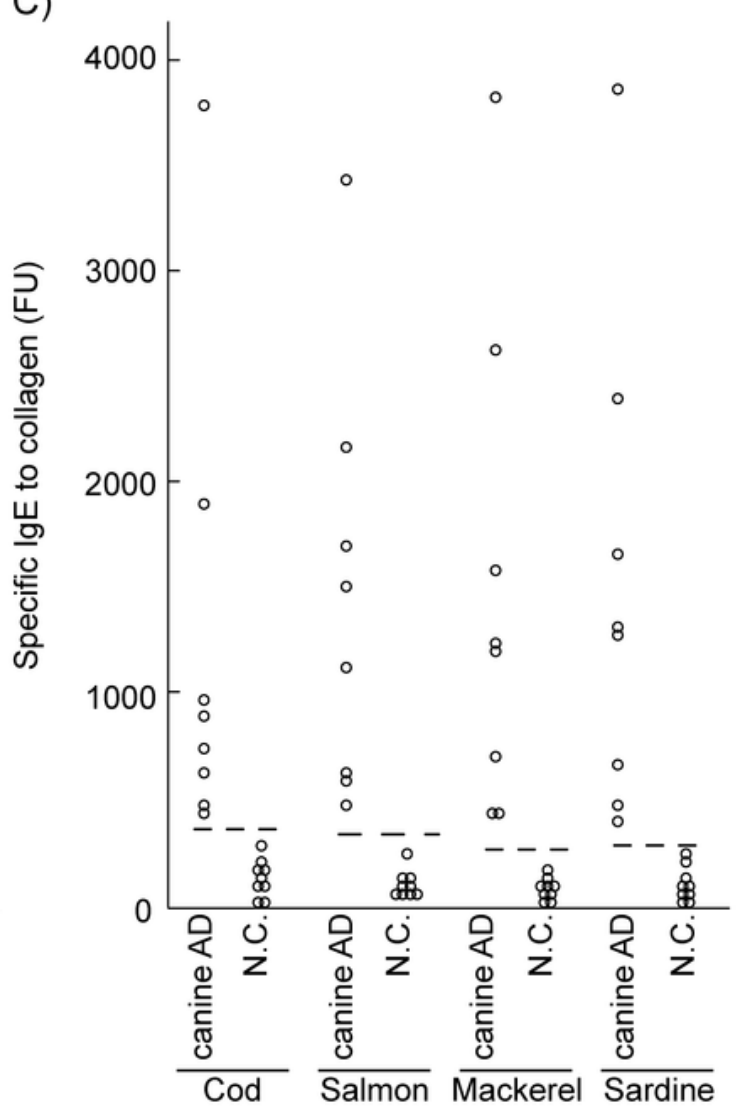

\section{Figure 2}

IgE reactivity to parvalbumin and collagen in atopic dogs with significantly elevated cod-specific $\lg \mathrm{E}$ levels. (A) IgE reactivity to cod parvalbumin and collagen in 36 dogs with IgE reactivity to crude cod extract was determined using diluted sera (1:10). The dotted line indicates the cutoff value, which was 
calculated using sera from 20 N.C. Based on the levels of specific IgE to parvalbumin and collagen in the N.C. (mean $\pm S D, 84 \pm 67$ and $74 \pm 25 \mathrm{FU}$, respectively), the cutoff values (mean $+3 \mathrm{SD}$ ) for specific lgE against parvalbumin and collagen were 286 and $149 \mathrm{FU}$, respectively. The levels of specific IgE to parvalbumin and collagen in atopic dogs were 299-1121 and 151-3774 FU, respectively. The blue circle represents IgE reactivity in dog no. 34. The red circle represents IgE reactivity in dog no. 128 . The levels of (B) Parvalbumin-specific IgE and (C) collagen-specific IgE was measured in the sera of six dogs with specific lgE to parvalbumin and eight dogs with specific lgE to collagen and in those of nine healthy dogs (N.C.). The cutoff values (mean + 3SD) of specific IgE were 379, 374, 351, and 372 FU for parvalbumin from cod, salmon, mackerel, and sardine, respectively, which are indicated by the dotted lines. The cutoff values (mean + 3SD) of specific lgE were 393, 310, 231, and 372 for collagen from cod, salmon, mackerel, and sardine, respectively. N.C., negative control. 


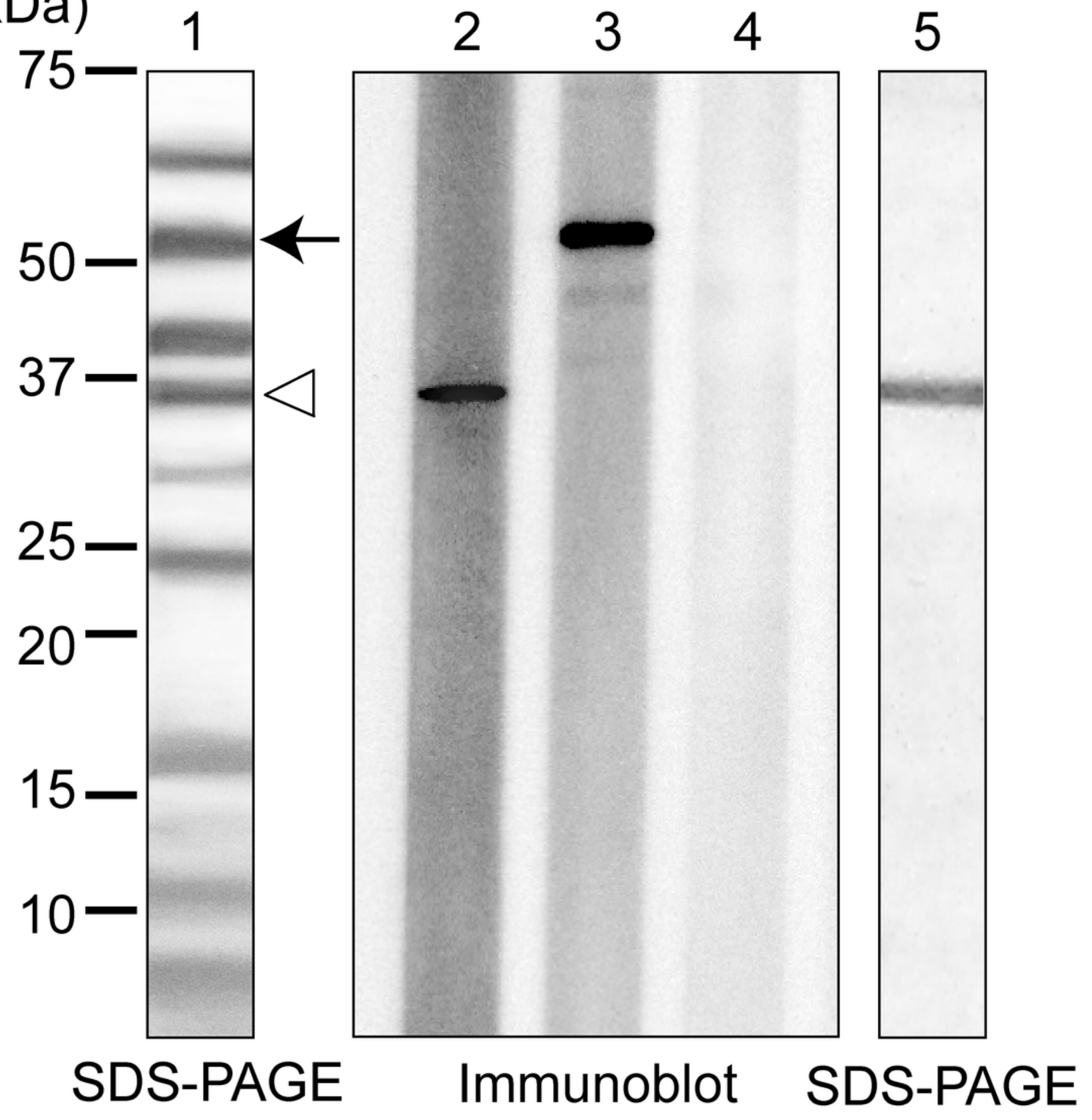

Figure 3

Immunoblotting for crude cod extracts. In the left column, the molecular standard is shown. Lanes 1 and 5 were stained by Coomassie brilliant blue R250. Lanes 2 and 3 depict immunoblotting in the sera samples of two atopic dogs (lane 2, dog no. 34; lane 3, dog no. 128); lane 4 represents the serum of a healthy dog used as negative control. The arrow and arrowhead next to lane 1 indicate the bands 
analyzed by liquid chromatography-tandem mass spectrometry, which corresponded to the band detected by immunoblotting (immunoblotting in lanes 2 and 3).

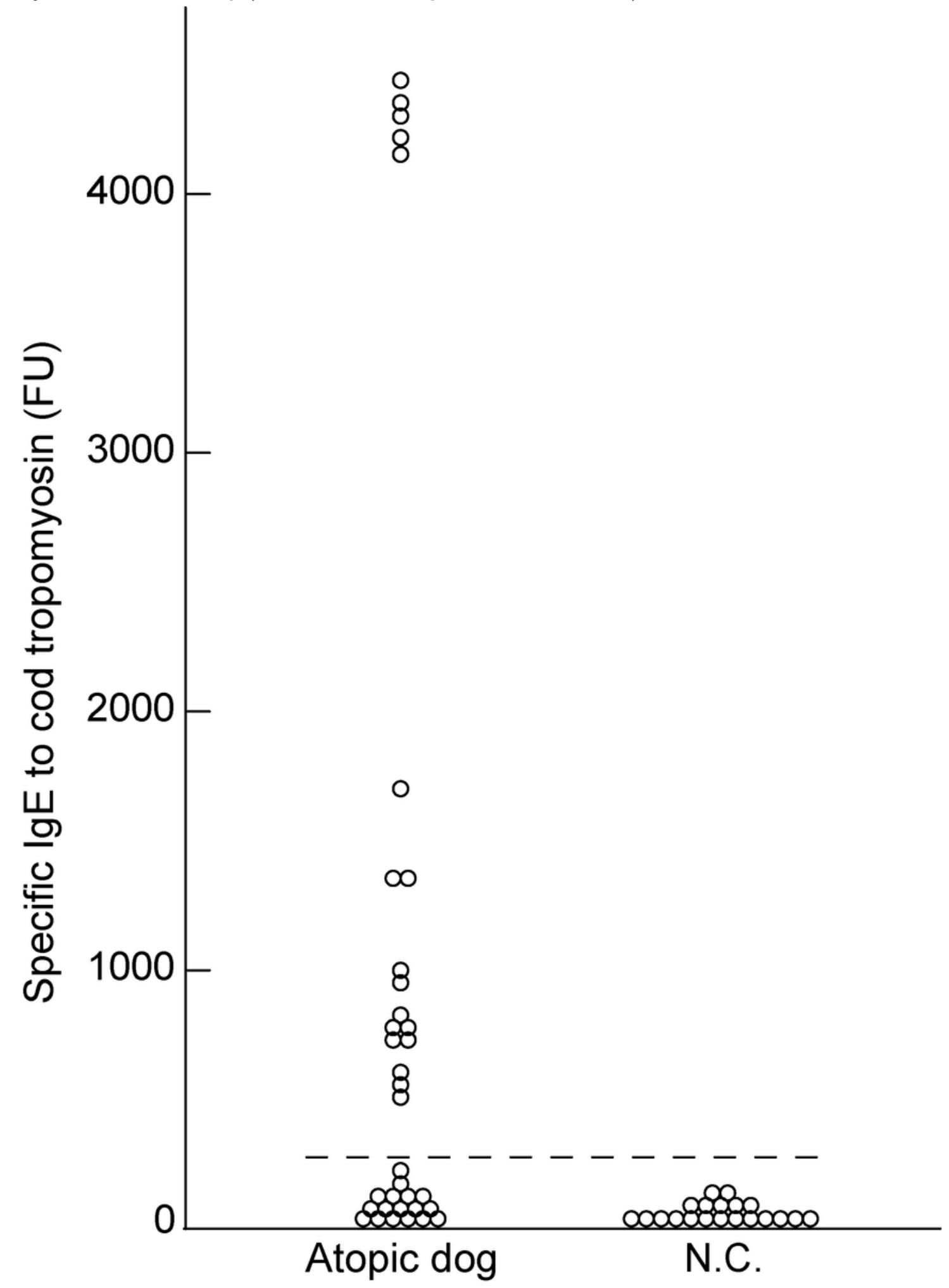

Figure 4

Reactivity to cod tropomyosin in dogs with specific lgE to crude cod extracts using ELISA. The cutoff value (dotted line) calculated from 20 negative control samples. Based on the levels of specific IgE to tropomyosin in the negative controls (mean \pm SD, $33 \pm 67 \mathrm{FU}$ ), the cutoff value (mean $+3 \mathrm{SD}$ ) of IgE 


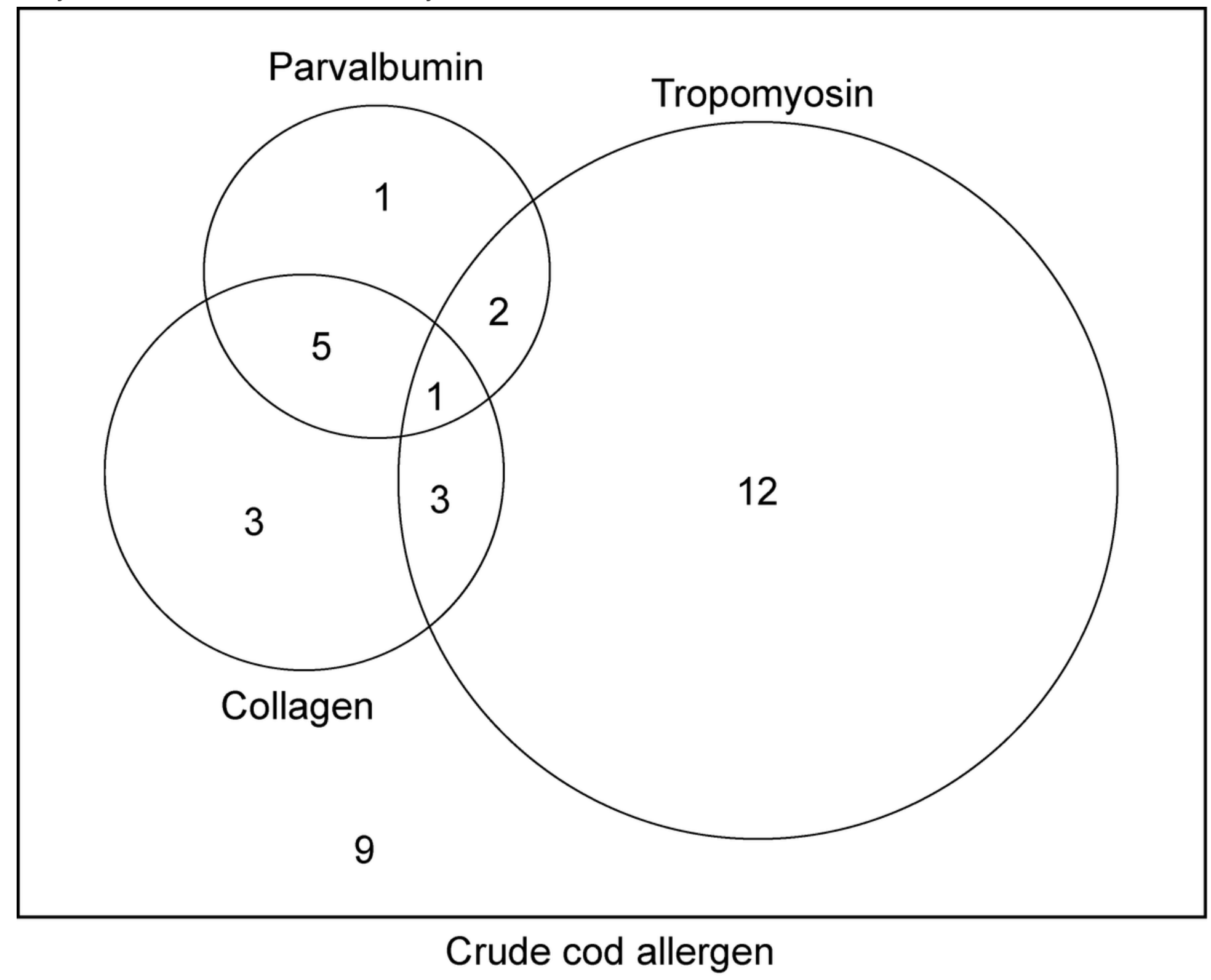

Figure 5

Venn diagram of the number of dogs harboring fish allergen-specific lgE.

\section{Supplementary Files}

This is a list of supplementary files associated with this preprint. Click to download.

- Additionalfile1.pdf 